\title{
RNAi, a new therapeutic strategy against viral infection
}

\author{
Fischer L. TAN, James Q. YIN* \\ Institute of Biophysics, Chinese Academy of Sciences, 15 Datun Road, Beijing 100101, China.
}

\begin{abstract}
RNA interference (RNAi) is an adaptive defense mechanism triggered by double-stranded RNA (dsRNA). It is a powerful reverse genetic tool that has been widely employed to silence gene expression in mammalian and human cells. RNAi-based gene therapies, especially in viral diseases have become more and more interesting and promising. Recently, small interfering RNA (siRNA) can be used to protect host from viral infection, inhibit the expression of viral antigen and accessory genes, control the transcription and replication of viral genome, hinder the assembly of viral particles, and display influences in virus-host interactions. In this review, we attempt to present recent progresses of this breakthrough technology in the above fields and summarize the possibilities of siRNA-based drugs.
\end{abstract}

Keywords: RNAi, siRNA, viral infection, gene therapy.

\section{INTRODUCTION}

RNA interference (RNAi), an ancient defense pathway, is a common denominator for the posttranscriptional gene silencing (PTGS) phenomenon observed in a variety of species such as plants, fungi and animals [1-3]. The introduction of long dsRNA into cells can effectively and specifically lead to the degradation of cognate mRNAs in a gene-dependent manner. This powerful technology has been widely employed to manipulate gene expression, elucidate signal pathways and to identify gene functions in a whole-genome scale. Researchers worldwide have used RNAi for basic research, and are now developing RNAi-based drugs for the prevention and treatment of human diseases such as viral infection, tumors and metabolic disorders [4-8].

Despite significant advances have been made in the therapy of viral diseases, current drugs and vaccines are restricted with many factors such as toxicity, complexity, cost and resistance [4]. RNAi is a self-defense mechanism of eukaryotic cells, which specially prevent infection evoked by viruses [5]. It can inhibit the expression of crucial viral proteins by targeting viral mRNA for degradation through cellular enzymes [9]. In fact, RNAi does work effectively as an antiviral agent in plants. This breakthrough technology emerges as a powerful tool to protect human cells from viral infection [10].

\footnotetext{
*Correspondence: James Q. YIN

Tel: +86-10-64888572, Fax: +86-10-64888572

E-mail: jqwyin@sun5.ibp.ac.cn
}

\section{MACHINERY OF RNAi}

Biochemical and genetic studies have revealed the molecular mechanismes, by which dsRNA causes the degradation of target messenger RNA [11]. RNAi includes two steps: initiation step and effector step [12]. In the initiation step, Dicer, a member of the RNase III family of ATP-dependent ribonucleases, binds with high affinity to dsRNA containing 2 nucleiotide (nt) 3' overhangs and chops long dsRNA (introduced directly or via a transgene or virus) into small interfering RNAs (siRNAs) duplexes. Generally, Dicer enzymes contain an N-terminal DEXH-box RNA helicase domain, a domain of unknown function (DUF283), a PAZ domain, two RIII domains and a dsRNA-bingding domain (dsRBD) [13]. Dicer can cleave dsRNA into siRNAs or microRNAs (miRNAs) from endogenous stem loop precursors [14]. Biochemical studies show that siRNAs are 21-23 nt dsRNA duplexes with 2-nt 3' overhangs, a 5'monophosphate and a 3'-hydroxyl group [15]. In the effector step, the siRNA duplexes are incorporated into RNA induced silencing complex (RISC). The phosphorylation of siRNA 5'-terminal is required to entry into RISC [16]. A helicase domain of RISC binds to one end of the duplex and unwinds the double-strand in an ATPdependent manner. The thermodynamic stability of the first few base pairs of siRNA can affect the ratio of RISC containing the antisense or sense strands of siRNAs [17]. Dicer with R2D2 (Dcr-2-associated protein) binds siRNA and facilitates its loading onto RISC [18]. The active RISC then targets the homologous transcript by base pairing interactions and cleaves the mRNA between the $10^{\text {th }}$ and 
$11^{\text {th }}$ nucleotide from the $5^{\prime}$ terminus of the siRNAs [ 16 , 19].

MicroRNAs (miRNAs) are endogenous $\sim 22 \mathrm{nt}$ RNAs that can play important regulatory roles in animals and plants by targeting mRNAs for cleavage or translational repression. miRNAs and endogenous siRNAs have a shared central biogenesis and can perform interchangeable biochemical functions. Therefore, the two classes of silencing RNAs cannot be distinguished by either their chemical composition or mechanism of action [20]. These short RNA species are produced by Dicer cleavage of long $(\sim 70 \mathrm{nt})$ endogenous precursors with imperfect hairpin RNA structure in animals. Mature miRNAs act as a translational repressor by partial base-pairing with 5' or 3' ends of mRNAs while miRNA completely complementary to its target mRNA (endogenous siRNA) can result in the degradation of cognate mRNA.

In addition, many other proteins such as eukaryotic translation initiation factor $2 \mathrm{C} 2$ (eIF2C2) and Argonaute proteins are likely to function in both pathways. Argonaute proteins are the key components of RISC. They are evolutionarily conserved with two distinguishing domains, PAZ and PIWI. The PIWI domain is restricted to Argonautes while the PAZ domain is shared with Dicer family proteins [21].

\section{SILENCING MECHANISMS OF RNAi}

Long dsRNA enables the effectively silencing of gene expression by presenting various siRNA sequences to target mRNA. Cells infected by viruses invariably produce dsRNA, but viruses can escape a profound cellular response. The dsRNA binds to dsRNA-bing proteins (dsRBPs), which have been shown to counteract the effects of Interferon (IFN) and the resultant dsRNA-acticated protein kinase (PKR) activation, and to suppress RNAi. Recently, it has become clear that 21-nucleotide siRNAs are so short that they cannot induce an interferon response in mammalian cells [22]. So, the antiviral effects of siRNAs have attracted more and more scientists to throw their interests into this field [23].

It has been shown that the inhibition of viral infection can be mediated by siRNA molecules that target viral mRNA for degradation. In comparison with other conventional drugs, siRNA have many advantages. First, it is much easier and more flexible to select target sites because target mRNA and siRNA are sequences-specific and complementary. For a given mRNA molecule, the inhibitory effects of siRNAs can be achieved by targeting different regions of target mRNA. Second, for gene silencing, only substoichiometric amount of siRNA is enough to drastically decrease homologous mRNA within $24 \mathrm{~h}$. Third, siRNAs can result in the degradation of cognate mRNA in cells of different species. Today, researchers are developing efficient siRNA delivery systems, which can help siRNAs enter efficiently into cells in nearly all organs. Fourth, siRNAs do not seem to adversely affect cell control mechanisms. The given length and high homology of siRNA to the target region of cognate transcription ensure the selective destruction of only interested transcript. siRNAs without suitable targets seem to remain inert within cells. This exclusive specificity without adverse side effects is the most attractive feature of RNAi as an antiviral approach. Fifth, siRNAs can silence gene stably. With the application of plasmid vectors and viral vectors, siRNAs can display their long-term biological effects [25]. Taken together, siRNAs synthesis in vivo or in vitro transfected into cultured cells or animals could result in the sequencespecific silencing of mRNA molecules. With the proof-ofconcept studies, siRNAs have been widely used as an alternate therapeutic strategy.

\section{RNAi APPLICATION TO VIRAL INFECTION}

Although there is still a long way to go before the RNAi technology will be employed to clinical application, researchers believe that RNAi will finally be used as a viable therapeutic alternative for various diseases in the near future $[6-8,26]$.

\section{RNAi prevents viral infection}

The ability of synthetic siRNAs to inhibit viral production was determined in cultured cells by introducing siRNAs into the cells before viral infection and then assaying virus titers in the culture supernatants at different times after infection [27]. In the first application of RNAi technology to prevent disease, researchers revealed that siRNA-directed inhibition of $F$ as gene expression could protect mice from antibody or concanavalin A-induced hepatitis [28]. Nowadays, potential siRNA-based prophylaxis has been widely used in research of viral diseases.

Influenza virus causes one of the most prevalent infections in humans. It is a RNA virus without any DNA intermediates during its entire life cycle and normally infects epithelial cells in the upper airway and the lungs. Introducing siRNAs into cultured cells and chicken embryos before virus infection was able to inhibit influenza virus production, especially those encoding NP, PA, and PB1. In addition, siRNA inhibitory effects also occurred in cells that had been infected with virus prior to siRNA introduction [27]. siRNAs can be administered by inhalation. It is convenient and able to achieve so high local siRNA concentration that epithelial cells can uptake sufficient siRNA. In order to achieve prophylactic effect, siRNAs may be administered prior to infection or during the early phase of infection. Thus, it is possible to achieve 
the prevention of influenza infection by inhalation of siRNA once per week or even less often [29].

Poliovirus, a RNA virus, is able to invade the nervous system and cause paralysis. The genome of poliovirus contains a long open reading frame encoding a single polyprotein, which can be divided into three major parts (P1-P3). The P1 region includes four structural proteins (VP1-VP4) that constitute the capsid. Gitlin et al revealed that RNAi effectively prevented viral infection in mammalian cells by using siRNAs against the poliovirus genome. Subsequently, they indicated that pretreatment of human and murine cells with siRNA duplexes to the poliovirus genome (capsid and 3D polymerase) could prevent plaque formation and result in a 100 -fold reduction of the progeny titer in one-step growth curves [9]. In these experiments, siRNAs promote clearance of the virus from most infected cells [10].

Hepatitis $\mathrm{C}$ virus (HCV) infection is a major cause of chronic liver disease, which can lead to the development of liver cirrhosis and hepatocellular carcinoma. Kapadia et al showed that NS3 and NS5B siRNAs could specifically inhibit HCV RNA replication and protein expression in Huh-7 cells. In their experiments, HCV RNA replication was inhibited within $2 \mathrm{~d}$ of siRNA transfection, and the effect lasted at least $6 \mathrm{~d}$ [30].

$\operatorname{AcNPV}$ (A. californica nucleopolyhedrovirus) is a member of baculoviruses family that naturally infects many different insect species. This virus has a large doublestranded DNA genome with over 135 open reading frames. iel gene is an early transcriptional activator essential for baculovirus replication. The introduction of dsRNA containing a sequence from iel or iel-siRNAs into Sf21 cells and living insects could protect them against baculovirus infection. Over $95 \%$ of Sf21 cells transfected with $5 \mu \mathrm{g}$ of dsRNA corresponding to the iel gene were not infected with recombinant baculovirus [31]. The inhibitory effects of siRNAs could be stable inside insect cells for over a week [32].

As it showed in above examples, siRNAs can protect against viral infection for several days with poliovirus, hepatitis $\mathrm{C}$ virus, influenza virus and baculoviruses. The stability of the siRNAs, combined with the efficient transfection observed in these experiments may demonstrate the application potential of RNAi to the prevention of viral infection.

\section{RNAi inhibits the expression of viral antigens}

Many viral proteins don't involve in viral RNA or protein synthesis, but take part in pre- or post-transcriptional aspects of the viral life cycle. A number of cellular membrane molecules may act as receptors for viruses. RNAi has widely been used in the inhibition of the expression of viral surface antigens in the research of viral diseases.

CD4 molecule is the main receptor for HIV-1 in HeLaderived cell line, Magi-CCR5, which expresses human CD4, CXCR4 and CCR5. To assess the effect of CD4 silencing on viral entry, $\beta$-galactosidase activity and syncytia formation were assessed as indicators of viral entry and infection, respectively. After Magi-CCR5 cells were infected with both R5 (BAL) macrophage-tropic and X4 (NL43) T cell-tropic strains of HIV-1 $60 \mathrm{~h}$ after transfection with siRNAs, results showed that the expression of CD4 decreased eight-fold and a four-fold reduction in viral entry. Few syncytia showed that there was little viral spread to neighboring uninfected cells. These investigations suggested that siRNA-directed CD4 silencing specifically inhibit HIV-1 infection [33].

CCR5, a human chemokine receptor protein, is a necessary coreceptor for infection by most strains of HIV-1. It is an important molecular for the infection process of CCR5-tropic strains of HIV-1 because the absence of CCR5 prevents HIV-1 infection to cells. When siRNAs against CCR5 were introduced into human peripheral blood T lymphocytes, the expression of potent CCR5-siRNA would result in up to 10-fold inhibition of CCR5 synthesis on the cell surface over a period of 2 weeks. The inhibition of CCR5 expression by siRNAs would protect lymphocytes from HIV-1 virus infection with a decrease in infected cells by 3 - to 7-fold [34].

Paramyxoviruses initiate infection by attaching to cell surface receptors and fusing viral and cell membranes. Three viral attachment proteins, glycoprotein (G) receptors, hemagglutinin-neuraminidase $(\mathrm{HN})$ and fusion (F) proteins direct membrane fusion (formation of syncytia). The siRNA knockdown of F resulted in complete loss of syncytium without G or SH synthesis [35]. Reciprocally, the abrogation of SH by siRNA had no effect on $\mathrm{F}$ or $\mathrm{G}$, and also had no appreciable effect on syncytium. Together, these results validated an essential role of $F$ and the dispensability of SH in cell fusion mediated by respiratory syncytial virus (RSV).

SARS-CoV (the SARS-associated coronavirus) has been classified as a novel member of the coronavirus family with a spike protein, which plays an important role in viral entry and pathogenesis [36]. Zhang et al showed that the DNA vector-driven siRNA against the spike protein of SARS-CoV could electively and specifically silence gene expression of the Spike protein in SARS-infected 293T cells [37]. Obviously, the suppression of expression of viral antigens by RNAi will be effective strategies for the therapy of viral infection.

RNAi suppresses the transcription of viral genome Viruses need to transcribe its genome after entering the 
host cells. In addition to using cellular factors, viruses can generate their transcriptional activators such as E6 and E7 proteins of human papillomaviruses (HPVs). HPV are small DNA viruses with a genome of approximately $8 \mathrm{~kb}$ and many subtypes. HPV16 is a main causative agent of cervical cancers and encodes the E6 and E7 oncogenes, which are essential for malignant transformation as well as maintenance of the tumor's malignant phenotype. E6 siRNAs were transfected into HPV16-related cervical cancer cells. Experiments demonstrated that E6 siRNAs were potent in the suppression of viral oncogene expression. Simultaneously, E6 siRNAs exhibited a potent growth inhibitory activity. Thus, anti-E6 siRNA may be used as a genespecific therapy for HPV-related cancers [38].

For RNA viruses, especially in retroviruses, gag, env and $p o l$ are essential for genome transcription. Avian Sarcoma Leucosis Virus (ASLV) is a positive-RNA virus, a subtype of RCAS. siRNAs matching two sequences in the gag gene of ASLV were introduced into cultured chicken DF-1 cells, one targeting the p19 matrix (MA) and another aiming at the p12 nucleocapsid (NC). After DF-1 cells were co-transfected by RCAS virus and siRNAs, the production of the RCAS virus in culture supernatants was dramatically reduced (90\%) $2 \mathrm{~d}$ after treatment [39]. Analysis of provirus formation documented that the antigag siRNAs could inhibit the transcription of RCAS [40]. Moreover, the inhibition of transcription by anti-gag siRNAs [41] and anti-env siRNAs [42] against HIV-1 virus genome was also observed in CD4-positive cells.

\section{RNAi blocks viral replication}

Once a virus has entered a cell, it needs to replicate its genome. It has been shown that RNAi is induced by virus replication in animals. Activation of RNA silencing is thought to be involved in the inhibition of viral replication.

Dengue (DEN) virus has a positive-polarity, singlestranded RNA genome of approximately $11 \mathrm{~kb}$ in length. It is a mosquito-borne member of the family Flaviviridae, genus Flavivirus, and can cause dengue fever and dengue hemorrhagic fever-dengue shock syndrome. Like other flaviviruses, DEN viruses generate detectable amounts of intracellular double-stranded RNA (dsRNA) as an intermediate of their replication. Adelman et al showed that C6/36 (Aedes albopictus) cells could stably be transformed with the plasmid containing dsRNA that was designed to transcribe an inverted-repeat RNA (irRNA) derived from the genome of dengue virus type 2 (DEN-2). Results demonstrated that $44 \%$ cell lines designed to express irRNA were resistant to DEN-2 infection, with more than 95\% of the cells showing no DEN-2 antigen accumulation [43]. DEN-2 genome RNA was completely failed to accumulate in FB 9.1 (a member of the DEN-2-resistant cell lines) cells after infection [44].

West nile virus (WNV) is an enveloped arbovirus with a single-stranded, 11-kb positive-polarity RNA genome that also belongs to the flavivirus genus of the Flaviviridae family of viruses. siRNAs specific for WNV capsid or NS5 ORF were constructed in pHH21 and then transfected into 293 T cells. Subsequently, the treated cells were infected with WNV. It was showed that WNV protein expression, genomic RNA synthesis, and infectious virus production were all dramatically reduced by siRNAs targeting these two distinct viral sequences [45].

The HBV genome is a double-stranded DNA molecule and generates four viral RNAs that encode the core protein/ $\mathrm{HBeAg}$ and polymerase-reverse transcriptase, $\mathrm{HBsAg}$, and $\mathrm{X}$ protein, respectively. Human hepatoma cells were co-transfected with HBV and siRNAs against HBVpregenome RNA. Experiments showed that the siRNA reduced the amount of HBV-pregenome RNA and led to decrease in the levels of replicative intermediates and viral protein. The results also indicated that siRNA-mediated gene silencing could inhibit HBV replication through suppression of viral RNA [46]. Recently, McCaffrey and colleagues developed the mouse model of HBV infection by using short hairpin RNAs (shRNAs) targeting HBV sequences, in which viral replication and the production of core antigen in hepatocytes were effectively inhibited [47].

RNAi has been widely used to target numerous viruses from diverse virus families. Highly effective inhibition of virus replication by RNAi has been achieved both in vitro and in vivo. With the development of RNAi, its application for viral infections will pave a new way towards clinical trials.

\section{RNAi silences viral accessory genes}

There are many accessory genes in viruses. These genes take part in the viral pathogenesis such as latency and persistence and regulate the expression of other genes. Silencing these genes should be good therapeutic strategies for the treatment of viral diseases.

Upon HIV-1 infection, viral RNA is introduced into the host cell cytoplasm in the form of a nucleoprotein complex. siRNA duplexes against several regions of the HIV-1 genome, including the viral long terminal repeat (LTR) and the accessory genes vif and nef were co-transfected with an HIV-1 molecular cloning into CD4-positive HeLa (Magi) cells. Compared with cells not transfected with siRNA duplexes, virus production was reduced 30fold to 50-fold by homologous siRNAs [48]. These results provided a therapeutic alternative for AIDS by siRNAmediated degradation.

Epstein-Barr virus (EBV) is a prototype gamma herpes 
virus with a dsDNA genome. It is the first human virus identified and is related to the pathogenesis of several malignancies, including Burkitt's and T cell lymphomas, Hodgkin's disease, breast and gastric carcinomas, and some AIDS-related lymphomas. Latent membrane protein-1 (LMP-1), encoded by EBV, is thought to be the only oncoprotein playing an essential role in cell transformation as well as nasopharyngeal carcinoma (NPC) metastasis. Li et al showed that the stable suppression of LMP-1 by shRNA plasmid significantly altered EBV-positive NPC cell (C666) motility, substratum adhesion, and transmembrane invasion ability [49].

Application of RNAi to the study of accessory genes provides a chance to learn more about molecular mechanisms underlying viral infection so that people could develop more effective drugs to treat viral diseases.

\section{RNAi hinders the assembly of viral particles}

Assembly of viral particles varies with different virus families. The formation of the virus caspid has been well studied. Many caspid proteins are involved in the assembly of virus particles including VP4 and matrix proteins.

Vesicular stomatitis virus (VSV) is a member of nonsegmented negative-stranded RNA viruses. VSV matrix gene $(\mathrm{M})$ mRNA was shown to produce three proteins: M1, M2 and M3. M protein(s) can inhibit host gene expression and cause apoptosis of the host cell [51]. In an attempt to determine which product of the $M$ gene mRNA is involved in apoptosis and cell-rounding, siRNAs targeting to the region of target mRNA that is unique to M1 and upstream of M2 and M3 were transfected into HEp-2 cells. Results showed that these siRNAs abrogated the expression of all three proteins, and the cell detachment was concomitantly inhibited [52].

Rotavirus is a non-enveloped viruses formed by three concentric layers of protein that enclose a double-stranded RNA (dsRNA) genome. Rotavirus can cause severe dehydrating diarrhea in infants and young children worldwide. VP4 is a spike-like structure protein. It has essential functions in the virus life-cycle, including the attachment of the virus particles to cell receptors and the penetration of the virions into the cell. Dector et al showed that a small interfering RNA corresponding to the $V P 4$ gene efficiently inhibited the synthesis of this protein in virus infected monkey kidney MA104 cells. A large proportion of infected cells had no detectable VP4 protein and the yield of viral progeny was reduced. Most of the virus particles purified from these cells were poorly infectious. The VP4 gene silencing was specific because the synthesis of VP4 gene from rotavirus strains with different target sequence was not affected. These findings offer a novel therapeutic approach for the assembly of viral particles [53].
siRNAs targeting to different viral protein genes can be used in the study of the assembly of viral particles. The knockdown of these genes will effectively prevent the viral infection.

\section{RNAi displays roles in virus-host interactions}

Viruses can control both viral and cellular gene expression while host cells can also protect themselves against virus infection through immune response. The suppression of these genes by siRNAs can be also used to address the role of such genes in host-virus interaction.

Bitko and his colleagues recently applied siRNAs-knockdown technology to determine the role of profilin in the RSV life cycle [54]. It was showed that profilin stimulated RSV transcription in vitro, but was not absolutely necessary for virus replication in the previous studies. The knockdown of profilin showed that it had a minor effect on viral transcription but strongly inhibited the formation of actin stress fibers in HEp-2 and L4 cells. In addition, it abrogated fusion in all cells tested. These results displayed an essential role of profilin in the gene expression of RSV and the actin formation of host cells.

Nodaviral infection can trigger an RNA silencing-based antiviral response (RSAR) in Drosophila RSAR, which is a rapid virus clearance in the absence of expression of a virus-encoded suppressor. Li et al showed that Drosophila RSAR was mediated by the RNA interference (RNAi) pathway, as the viral suppressor of RSAR inhibited experimental RNAi initiated by exogenous double-stranded RNA [55]. Specific RNA silencing was induced by IFN. Antagonist proteins encoded by viruses are suppressors of the Drosophila S2 cells in response to viral RNA replication. Furthermore, this mosquito antiviral immunity is RISC dependent and sensitive to suppression evoked by the $\mathrm{B} 2$ protein encoded with either FHV or nodamura virus (NoV).

$\mathrm{HCV}$ belongs to the family Flaviviridae with a positive RNA genome. To determine whether HCV expression is interfered with RNAi, siRNAs against cellular (LaminA/C) and viral (HCV) RNAs were introduced into Huh-7.5 cells containing replicating HCV. Both cellular and viral RNAs were effectively silenced [56]. The efficiency of silencing $\operatorname{lamin} \mathrm{A} / \mathrm{C}$ expression was similar either in the presence or absence of replicating HCV RNAs. Simultaneously, strand-specific probing for HCV RNAs revealed that siRNAs reduced the levels of both positive and negative strand RNA [26].

The study of virus-host interactions can promote the understanding of RNAi machinery as well as the application of RNAi in the viral diseases, which will extremely draw more researchers to dedicate to it. 


\section{CONCLUSIONS}

Virus infection is a severe public health problem with significant personal, social, and economical consequences. More effective approaches are urgently needed to treat viral infection. Recent works have shown that the application of RNAi can inhibit viral infection by targeting viral or cellular genes. RNAi is considered as a gene-specific therapeutic option for controlling viral infection. However, the control of viral infection is difficult and complex because of the limited effectiveness of existing anti-viral agents and the high speed of mutation rate of the viral genome [57]. Careful assessments are required for the potential of RNAi as a gene therapy approach for controlling viral infection. The challenge of successful siRNAbased drugs in the near future is to develop efficient and safe means to deliver siRNAs into cells interested.

RNAi has been used in large-scale, genome-wide screens. Scientists have developed many RNAi libraries to study the function of genes in nematodes [58] and human [59], which can greatly facilitate the identification of drug targets against viral infection [60]. With the advent of RNAi library in mammals and the refinement of techniques to silence gene, siRNA-based drugs will surely make great advances in the prevention and treatment of viral diseases.

\section{REFERNCES}

1 Fire A, Xu S, Montgomery MK, et al. Potent and specific genetics interference by double-stranded RNA in Caenorhabitis elegans. Nature 1998; 391: 806-11.

2 Jorgensen R. Altered gene expression in plants due to trans interactions between homologous genes. Trends Biotechnol 1990; 8: 340-4.

3 Romano N, Macino G. Quelling: transient inactivation of gene expression in Neurospora crassa by transformation with homologous sequences. Mol Microbiol 1992; 6: 3343-53.

4 Dave RS, Pomerantz RJ. RNA interference: on the to an to alternate therapeutic strategy. Rev Med Virol 2003; 13: 373-85.

5 Plasterk RA. RNA silencing: the genome's immune system. Science, 2002; 296: 1263-5.

6 Yin JQ, Wan Y. siRNA-mediated gene regulation system. Int J Mol Med 2002; 10:355-65.

7 Yin JQ, Cao JS, Shao RG, et al. SiRNA agents inhibit oncogene expression and attenuate human tumor cell growth. J Exp Ther Onc 2003; 3:1-11.

8 Liu TG, Yin JQ, Shang BY, et al. Silencing of hdm2 oncogene by siRNA inhibits p53-dependent human breast cancer. Cancer Gene Therapy (In Press).

9 Gitlin L, Myers CP, Andino R. Short interfering RNA confers intracellular antiviral immunity in human cells. Nature 2002; 418: 430-4.

10 Saleh MC, VanRij RP, Andino R. RNA silencing in viral infections: insights from poliovirus. Virus Res 2004; 102:11-7.

11 Dykxhoorn DM, Novina CD, Sharp PA. Killing the messenger: short RNAs that silence gene expression. Nat Rev Mol Cell Biol 2003; 4:457-67.

12 Hutvagner G, Zamore PD. RNAi: nature abhors a double-strand.
Curr Opin Genet Dev 2002; 12:225-32.

13 Carmell MA, Hannon GJ. RNase III enzymes and the initiation of gene silencing. Nat Struct Mol Biol 2004; 11:214-8.

14 Lee YS, Nakahara K, Pham JW, et al. Distinct roles for drosophila Dicer-1 and Dicer-2 in the siRNA/miRNA silencing pathways. Cell 2004; 117:69-81.

15 Elbashir SM, Lendeckel W, Tuschl T. RNA interference is mediated by 21- and 22- nucleotide RNAs. Genes Dev 2001; 15:188200.

16 Khvorova A, Reynolds A, Jayasena1 SD. Functional siRNAs and miRNAs exhibit strand bias. Cell 2003; 115:209-16.

17 Drosett Y, Tuschl T. siRNAs: applications in functional genomics and potential as therapeutics. Nat Rev Drug Discov 2004; 3: 319-29.

18 Pham JW, Pellino JL, Lee YS, Carthew RW, Sontheimer EJ. A Dicer-2-dependent $80 \mathrm{~S}$ complex cleaves targeted mRNAs during RNAi in Drosophila. Cell 2004; 117:83-94.

19 Ahlquist P. RNA-dependent RNA polymerases, viruses, and RNA Silencing. Science 2002; 296:1270-3.

20 Bartel DP. MicroRNAs: genomics, biogenesis, mechanism and function. Cell 2004; 116:281-97.

21 Song JJ, Liu J, Tolia NH, et al. The crystal structure of the Argonaute2 PAZ domain reveals an RNA binding motif in RNAi effector complexes. Nat Struct Biol 2003; 10:1026-8.

22 Semizarov D, Frost L, Sarthy A, et al. Specificity of short interfering RNA determined through gene expression signatures. Proc Natl Acad Sci U S A 2003; 100:6347-52.

23 Lichner Z, Silhavy D, Burgyan J. Double-stranded RNA-binding proteins proteins could suppress RNA interference-mediated antiviral defences. J Gen Virol 2003; 84:975-80.

24 Ding SW, Li H, Lu R, Li F, Li WX. RNA silencing: a conserved antiviral immunity of plants and animals. Virus Res 2004; 102: 109-15.

$25 \mathrm{Li} \mathrm{H}, \mathrm{Li}$ WX, Ding SW. Induction and suppression of RNA silencing by an animal virus. Science 2002; 296:1319-21.

26 Wilson JA, Jayasena S, Khvorova A, et al. RNA interference blocks gene expression and RNA synthesis from hepatitis C replicons propagated in human liver cells. Proc Natl Acad Sci U S A, 2003; 100:2783-8.

27 Ge Q, McManus MT, Nguyen T, et al. RNA interference of influenza virus production by directly targeting mRNA for degradation and indirectly inhibiting all viral RNA transcription. Proc Natl Acad Sci U S A 2003; 100:2718-23.

28 Song E, Lee SK, Wang J, et al. RNA interference targeting Fas protects mice from fulminant hepatitis. Nat Med 2003; 9:347-51.

$29 \mathrm{Ge}$ Q, Eisen HN, Chen J. Use of siRNAs to prevent and treat influenza virus infection. Virus Res 2004, 102:37-42.

30 Kapadia SB, Brideau-Andersen A, Chisari FV. Interference of hepatitis $\mathrm{C}$ virus RNA replication by short interfering RNAs. Proc Natl Acad Sci U S A 2003; 100:2014-8.

31 Valdes VJ, Sampieri A, Sepulveda J, Vaca L. Using doublestranded RNA to prevent in vitro and in vivo viral infections by recombinant baculovirus. J Biol Chem 2003; 278:19317-24.

32 Flores-Jasso CF, Valdes VJ, Sampieri A, et al. Silencing structural and nonstructural genes in baculovirus by RNA interference. Virus Res 2004; 102:75-84.

33 Novina CD, Murray MF, Dykxhoorn DM, et al. siRNA-directed inhibition of HIV-1 infection. Nat Med 2002; 8:681-86.

34 Qin XF, An DS, Chen IS, Baltimore D. Inhibiting HIV-1 infec- 
tion in human $\mathrm{T}$ cells by lentiviral-mediated delivery of small interfering RNA against CCR5. Proc Natl Acad Sci U S A 2003, 100: $183-8$

35 Bitko V, Barik S. Phenotypic silencing of cytoplasmic genes using sequence-specific double-stranded short interfering RNA and its application in the reverse genetics of wild type negative-strand RNA viruses. BMC Microbiol 2001; 1:34.

36 Drosten C, Gunther S, Preiser W, et al. Identification of a novel coronavirus in patients with severe acute respiratory syndrome. N Engl J Med 2003; 348:1967-76.

37 Zhang Y, Li T, Fu L, et al. Silencing SARS-CoV Spike protein expression in cultured cells by RNA interference. FEBS Lett 2004; 560:141-6.

38 Yoshinouchi M, Yamada T, Kizaki M, et al. In vitro and in vivo growth suppression of human papillomavirus 16-positive cervical cancer cells by E6 siRNA. Mol Ther 2003; 8:762-8.

39 Hu WY, Myers CP, Kilzer JM, Pfaff SL, Bushman FD. Inhibition of retroviral pathogenesis by RNA interference. Curr Biol 2002; 12:1301-11.

$40 \mathrm{Hu}$ WY, Bushman FD, Siva AC. RNA interference against retroviruses. Virus Res 2004; 102:59-64.

41 Capodici J, Kariko K, Weissman D. Inhibition of HIV-1 infection by small interfering RNA-mediated RNA interference. J Immunol 2002; 169:5196-201.

42 Park WS, Miyano-Kurosaki N, Hayafune M, et al. Prevention of HIV-1 infection in human peripheral blood mononuclear cells by specific RNA interference. Nucleic Acids Res 2002; 30:4830-5.

43. Adelman ZN, Sanchez-Vargas I, Travanty EA, et al. RNA silencing of dengue virus type 2 replication in transformed C6/36 mosquito cells transcribing an inverted-repeat RNA derived from the virus genome. J Virol 2002; 76:12925-33.

44 Sanchez-Vargas I, Travanty EA, Keene KM, et al. RNA interference, arthropod-borne viruses, and mosquitoes. Virus Res 2004; 102:65-74.

45 McCown M, Diamond MS, Pekosz A. The utility of siRNA transcripts produced by RNA polymerase I in down regulating viral gene expression and replication of negative- and positivestrand RNA viruses. Virology 2003; 313:514-24.

46 Hamasaki K, Nakao K, Matsumoto K, et al. Short interfering RNA-directed inhibition of hepatitis B virus replication. FEBS Lett 2003, 543:51-4.
47 McCaffrey AP, Nakai H, Pandey K, et al. Inhibition of hepatitis $B$ virus in mice by RNA interference. Nat Biotechnol 2003; 21: 639-44.

48 Jacque JM, Triques K, Stevenson M. Modulation of HIV-1 replication by RNA interference. Nature 2002; 418:435-8.

49 Li XP, Li G, Peng Y, Kung HF, Lin MC. Suppression of EpsteinBarr virus-encoded latent membrane protein-1 by RNA interference inhibits the metastatic potential of nasopharyngeal carcinoma cells. Biochem Biophys Res Commun 2004; 315:212-8.

50 Tilly G, Chapuis J, Vilette D, Laude H, Vilotte JL. Efficient and specific down-regulation of prion protein expression by RNAi. Biochem and Biophys Res Commun 2003; 305:548-51.

51 Kopecky SA, Lyles DS. The cell-rounding activity of the vesicular stomatitis virus matrix protein is due to the induction of cell death. J Virol 2003; 77:5524-8.

52 Barik S. Control of nonsegmented negative-strand RNA virus replication by siRNA. Virus Res 2004; 102:27-35.

53. Dector MA, Romero P, Lopez S, Arias CF. Rotavirus gene silencing by small interfering RNAs. EMBO Rep 2002; 3:117580.

54 Bitko V, Oldenburg A, Garmon NE, Barik S. Profilin is required for viral morphogenesis, syncytium formation, and cell-specific stress fiber induction by respiratory syncytial virus. BMC Microbiol 2003; 3:9.

$55 \mathrm{Li} \mathrm{WX}$, Li H, Lu R, et al. Interferon antagonist proteins of influenza and vaccinia viruses are suppressors of RNA silencing. Proc Natl Acad Sci U S A 2003; 101:1350-55.

56 Randall G, Grakoui A, Rice CM. Clearance of replicating hepatitis $\mathrm{C}$ virus replicon RNAs in cell culture by small interfering RNAs. Proc Natl Acad Sci U S A 2003; 100:235-40.

57 Lee NS, Rossi JJ. Control of HIV-1 replication by RNA interference. Virus Res 2004; 102:53-8.

58 Kamath RS, Fraser AG, Dong Y, et al. Systematic functional analysis of the Caenorhabditis elegans genome using RNAi. Nature 2003; 421:231-7.

59 Paddison PJ, Silva JM, Conklin DS, et al. A resource for largescale RNA-interference-based screens in mammals. Nature 2004; 428:427-31.

60 Berns K, Hijmans EM, Mullenders J, et al. A large-scale RNAi screen in human cells identifies new components of the p53 pathway. Nature 2004; 428: 431-7. 